\title{
Influenza Surveillance Among Hospitalized Children in Shenzhen Children's Hospital, 2012-2016
}

\author{
Zhi-wei Lu, He-ping Wang, Yue-jie Zheng* and Hai-xia Zhao \\ Department of Respiratory Diseases, Shenzhen Children's Hospital, China
}

*Corresponding author: Yuejie Zheng, Department of Respiratory Diseases, Shenzhen Children's Hospital, Yitian Rd, Shenzhen

518026, China, Email: yuejiez@sina.com

\begin{tabular}{l} 
ARTICLE INFO \\
\hline Received: 慧 March 27, 2019 \\
Published: 輩 April 03, 2019 \\
\hline Citation: Zhi-wei Lu, He-ping Wang, \\
Yue-jie Zheng, Hai-xia Zhao. Influenza \\
Surveillance Among Hospitalized Chil- \\
dren in Shenzhen Children's Hospital, \\
2012-2016. Biomed J Sci \& Tech Res \\
16(4)-2019. BJSTR. MS.ID.002898.
\end{tabular}

ABSTRACT

Objective: Influenza is one of the most important causes of hospitalization and death in children. The current monitoring of influenza in China is mainly conducted on outpatients with mild signs of influenza. To fill the gap in monitoring inpatients of severe influenza, we carried out routine influenza surveillance in hospitalized children with lower respiratory infection (LRI) in Shenzhen Children's Hospital.

Methods: From January 2012 to December 2016, routine influenza virus testing was performed in inpatients with acute LRI. Recorded data was then used to explore epidemiological and seasonal characteristics.

Results: The rate of positive influenza virus identification was $9.50 \%, 2.47 \%, 3.75 \%$, $2.62 \%$ and $4.84 \%$ in 2012, 2013, 2014, 2015 and 2016 respectively. In the spring, summer, autumn and winter, the positive detection rate of influenza virus was $4.76 \%, 3.53 \%$, $2.28 \%$ and $4.63 \%$ respectively. Among recorded 7 died cases (4 males and 3 females), who aged from 10 months to 4.3 years, and three of them had underlying disease. Of the deaths, 3 were due to acute necrotizing encephalopathy and 2 suffered from severe sepsis which were caused by Streptococcus pneumoniae infection.

Conclusion: In Shenzhen, the high incidence of influenza is identified in the spring. After the global pandemic of influenza A H1N1, the flu remains an important cause of childhood hospitalization and death. The main causes of death in children are influenza-associated acute necrotizing encephalopathy and secondary severe bacterial infection.

\section{Introduction}

Influenza is an acute respiratory tract infection caused by an influenza virus. Highly contagious, it spreads rapidly and widely and exhibits seasonal changes in infection rates. The influenza virus is one of the major causes of acute respiratory tract infection in the world. In non-pandemic situations, annual flu epidemics are not only serious threats to susceptible people, but also have important effects on public health [1]. Children are the most susceptible group and the most likely to spread the infection. During annual flu seasons, the illness mainly occurs in children, especially schoolchildren aged 5-17 [2]. Since the 2009 H1N1 pandemic, there have been a large number of influenza A (H1N1)-related deaths reported [3-6]. China is recognized as a flu-prone country. Herein, influenza surveillance among Chinese pediatric patients will contribute not only to the understanding of the trends of influenza in China but also to the understanding of the clinical characteristics of children with flu infection. Importantly, it will provides scientific evidence to support further studies. Current surveillance projects in China focus mainly on outpatients with mild flu-like symptoms, and there is a dearth of data on severe and critically ill hospitalized children. Since January 2012, we have conducted a routine 2009 H1N1 influenza A testing on children hospitalized in Shenzhen Children's Hospital for lower respiratory tract infection.

\section{Materials and Methods \\ Case Cohort}

A total of 28,883 children were selected from 2012-2016. They were hospitalized in Shenzhen Children's Hospital due to lower respiratory tract infections. 18,216 were male and 10,667 female. They ranged in age from 1 month to 14 years. 


\section{Methods}

After admission, a throat swab specimen was collected from each child and sent to the virus laboratory for influenza $A$ virus antigen detection. The colloidal gold test was used per the manufacturer's instructions. A color change in a specific zone was considered positive. All deaths were tested for influenza A nucleic acid.

\section{Statistical Analysis}

SPSS 16.0 software was used for data analysis. Descriptive statistics were used to analyze influenza epidemic data. Age distribution was analyzed. Count data are expressed in (\%). Intergroup comparison was performed using Chi-square test $(\chi 2)$ and $\mathrm{P}<0.05$ was considered statistically significant.

\section{Results}

\section{Detection of Respiratory Tract Influenza A Virus}

From January 2012 to December 2016, a total of 28,883 specimens were tested and 1117 (3.87\%) were found to be positive. The detection rates in different seasons (spring, summer, autumn, and winter) were $4.76 \%, 3.53 \%, 2.28 \%$, and $4.63 \%$ respectively. The positive detection rates in 2012, 2013, 2014, 2015, and 2016 were $9.50 \%, 2.47 \%, 3.75 \%, 2.62 \%$, and $4.84 \%$ respectively.

\section{Time Distribution}

From January 2012 to December 2016, the positive detection rate of influenza virus in Shenzhen Children's Hospital peaked twice. The first peak occurred in March-August 2012, and the second in March 2016. Sporadic cases were observed in April 2013, February 2014, June 2014, January 2015, and June 2015 (Figure 1).

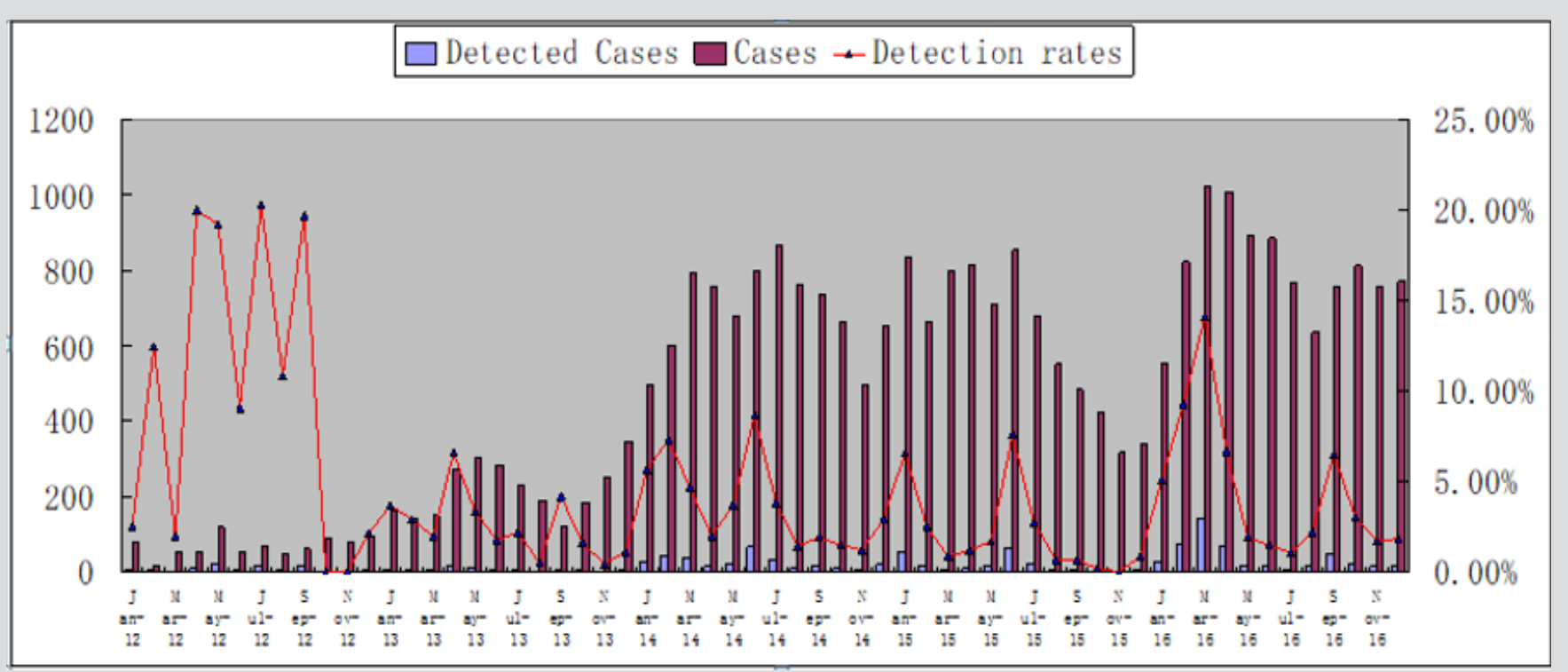

Figure 1: Time distribution of H1N1 influenza A in Shenzhen, 2012-2016.

\section{Gender Distribution}

Among the positive cases, 750 were male (750/18216, 4.12\%) and 367 were female $(367 / 10667,3.44 \%)$. The chi-square test $(\chi 2=8.287, \mathrm{P}=0.004)$ showed the male group to have a significantly higher positive rate than the female group.

\section{Age Distribution}

The following were the detection rates for different age groups: $3.48 \%(179 / 5143)$ for age 0-6 months, 3.25\% (308/9473) for 6-12 months, $4.48 \%$ (321/7167) for $1-3$ years, $4.79 \%$ (233/4868) for 4-6 years and 3.41\% (76/2232) for 7-14 years. The 4-6-yearold group had a significantly higher detection rate than the $0-6$ months, 6-12 months, or 7-14-year-old groups (chi-square values and $\mathrm{P}$ values were $10.8, \mathrm{P}=0.001 ; 20.8, \mathrm{P}=0.000$, and $8.47, \mathrm{P}=0.004$, respectively).

\section{Influenza-associated Deaths in Critically ILL Children}

From 2012-2016, a total of 8 children were admitted to the intensive care unit due to the flu infection, and 7 of them died. These children ranged in age from 10 months to 4.3 years. 4 were male and 3 were female. The main clinical manifestations included fever, cough, shortness of breath, and progressive dyspnea. Three cases had convulsions, persistent seizures, coma, or other neurological manifestations. One death occurred in May 2012, a 3-year-old boy who died from influenza A-related encephalopathy, brain herniation and pneumonia. The other 6 deaths occurred in March and May 2016. Three of these patients developed influenza-associated acute necrotizing encephalopathy. Three cases had underlying diseases, 1 each of acute lymphoblastic leukemia (with chemotherapy-induced myelosuppression), familial necrotizing encephalopathy (with an autosome dominant form), and nephrotic syndrome. The other two 
cases without underlying disease developed a complication of flu: severe sepsis caused by invasive streptococcus pneumoniae. All deaths had been infected by 2009 H1N1 influenza A virus.

\section{Discussion}

H1N1 influenza A virus is still a common cause of respiratory tract infection that seriously threatens child health. Most children have mild illness and only show flu-like symptoms such as fever and cough. Some children, however, are more likely to develop flu complications, such as severe pneumonia and neurological complications, which could be fatal [7]. Because influenza surveillance in China has mainly focused on outpatients at sentinel hospitals, it is difficult to extract data on critical illness and influenza-related death. Therefore, it is necessary to conduct flu surveillance among hospitalized children, especially critically ill children. According to the data of positive detection rate and season distribution, H1N1 influenza A viruses are most common during spring and summer. After flu season peaked (spring, summer and autumn) in 2012, flu activity started to drop although there were still sporadic cases observed in spring and summer. Since October 2012, the rate of H1N1 influenza A infection has decreased significantly. One reason for this decline could be that the majority of people have gained protective antibodies to H1N1 after their infection in 2009 H1N1 pandemic. Another important reason could be the flu vaccines recommended for susceptible people including preschool and schoolchildren [8-9].

Previous studies have shown that the flu in northern China mainly occurs in winter, but the flu in the south has no seasonality and can last a whole year. There could be 2-3 flu peaks each year, one in winter and spring, and the other in summer and autumn. However, the flu activity peaked mainly in spring during our 5-year surveillance. Based on the data in our hospital, H1N1 influenza A positive rate was highest among children 4-6 years old. A similar age distribution has been observed among children in Hebei Province, which showed the highest detection rate in the 4-6 and the 7-14-year-old groups. Children in these age groups are mainly preschool and school-age children. They lack immunity to influenza and tendency to stay in crowded places for long periods of time are two main factors that cause the flu to spread among these high-risk population. Therefore, it is of great significance to develop strategies for the prevention and control of influenza. Schoolteachers and staff need to increase their knowledge and awareness of importance of flu prevention and improve their abilities to deal with emergency situations. Parents also need to be educated about preventing the spread of the flu.

Pneumonia is the most common complication of influenza. It can be primary viral pneumonia or secondary bacterial infection. A few severe cases can quickly deteriorate in a short term, with respiratory failure, respiratory distress syndrome and even neurological complications such as encephalitis or encephalopathy. These severe complications are the main causes of influenza- induced death [10-12]. Based on the data from multi-center studies in China, 49 (6\%) hospitalized children developed encephalopathy and encephalitis as the complications of 2009 H1N1 Influenza and 13 deaths $(13 / 49,26.5 \%)$ occurred. It has been suggested that H1N1 flu-induced encephalopathy or encephalitis is also one of the major causes of child deaths in our country [13]. Of the 7 deaths observed in our surveillance, 3 were children who died from acute necrotizing encephalopathy and 2 cases died from secondary bacterial infection, which is basically consistent with domestic report. It is suggested that H1N1 flu can cause serious harm during both pandemic and non-endemic periods and herein should be given more attention.

Early recognized risk factors for influenza complications are: children under 5 years of age and the elderly over 65 years of age, obesity, pregnant women and perinatal women, previous underlying disease. Subsequent studies have shown that nosocomial influenza a virus infection, delayed treatment, and admission to the PICU were independent risk factors for influenza death [14-15].A recent meta-analysis indicated that the previously recognized risk factors for influenza-related complications, including pregnancy and ethnicity, had a low level of evidence and could not be confirmed as high risk factors, requiring further confirmation and research [16]. In the current study, 3 out of the 7 deaths were associated with underlying diseases, which suggested that patients with chronic diseases or cancers were more likely develop critical illness. In addition, 6 out of the 7 deaths in this study occurred in the spring of 2016. Given the cyclical nature of influenza epidemics, six years after 2009 H1N1 pandemic, H1N1 flu activity peaked once again in Shenzhen, causing recorded fatalities. The number of deaths reported in current study is consistent with the number from 2009. Currently, the exact timing and area of flu epidemics are not predictable. Therefore, better understanding of the cyclical trends of the flu epidemic has become very important for disease prediction.

\section{Conclusion}

In Shenzhen, the high incidence of influenza is identified in the spring. After the global pandemic of influenza A H1N1, the flu remains an important cause of childhood hospitalization and death. The main causes of death in children are influenza-associated acute necrotizing encephalopathy and secondary severe bacterial infection.

\section{Acknowledgement}

This work was supported by Sanming Project of Medicine in Shenzhen (SZSM201512030),Key Medical Disciplines Building Project of Shenzhen (SZXJ2017005), and Shenzhen public service platform for clinical drug trials (20151964).

\section{References}

1. Mistry RD, Fischer JB, Prasad PA, Coffin SE, Alpern ER, et al. (2014) Severe complications in Influenza-like illnesses. Pediatrics 134(3): e684-690. 
2. Lafond KE, Nair H, Rasooly MH, Valente F, Booy R, et al. (2016) Global Role and Burden of Influenza in Pediatric Respiratory Hospitalizations, 1982-2012: A Systematic Analysis. PLoS Med 13(6): e1002060.

3. Kumar S, Havens PL, Chusid MJ, Willoughby RE Jr, Simpson P, et al. (2010) Clinical and epidemiologic characteristics of children hospitalized with 2009 pandemic H1N1 influenza A infection. Pediatr Infect Dis J 29(7): 591-594.

4. Libster R, Jimena Bugna J, Coviello S, Hijano DR, Dunaiewsky M, et al. (2010) Pediatric hospitalizations associated with 2009 pandemic influenza A (H1N1) in Argentina. N Engl J Med 362(1): 45-55.

5. CDC (2009) Surveillance for pediatric deaths associated with 2009 pandemic influenza A (H1N1) virus infection--United States, AprilAugust 2009. MMWR Morb Mortal Wkly Rep 58(34): 941-947.

6. (2012) Centers for Disease Control and Prevention(CDC) Severe influenza among children and young adults with neurologic and neurodevelopmental conditions-Ohio,201l. MMWR Morb Mortal Wkly Rep 60 (51-52): 1729-1733.

7. Halasa NB (2010) Update on the 2009 pandemic influenza A H1N1 in children. Curr Opin Pediatr 22(1): 83-87.

8. Huang DT, Shao PL, Huang KC, Lu CY, Wang JR, et al. (2011) Serologic status for pandemic (H1N1) 2009 virus, Taiwan. Emerg Infect Dis 17(1): 76-78.

9. Griffin MR, Monto AS, Belongia EA, Treanor JJ, Chen Q et al. (2011) Effectiveness of Non-Adjuvanted Pandemic Influenza A Vaccines for Preventing Pandemic Influenza Acute Respiratory Illness Visits in 4 U.S. Communities. PLoS One 6(8): e23085.

\section{ISSN: 2574-1241}

DOI: 10.26717/BJSTR.2019.16.002898

Yuejie Zheng. Biomed J Sci \& Tech Res

(c) This work is licensed under Creative

Submission Link: https://biomedres.us/submit-manuscript.php
10. Ekstrand JJ, Herbener A, Rawlings J (2010) Heightened neurologic complications in children with pandemic H1N1 influenza. Ann Neurol 68(5): 762-766

11. Araujo R, Gouveia P, Fineza I (2016) Bilateral thalamic lesions in acute necrotizing encephalopathy due to H1N1 infection . Pediatr Neurol 65: 96-97.

12. Okuno H, Yahata Y, Tanaka-Taya K, Arai S, Satoh H, et al. (2018) Characteristics and Outcomes of Influenza-Associated Encephalopathy Cases Among Children and Adults in Japan, 2010-2015.Clin Infect Dis 66(12): 1831-1837

13. Liu XX, Qin G, Li X, Zhang J, Zhao K, et al. (2017) Excess mortality associated with influenza after the $2009 \mathrm{H} 1 \mathrm{~N} 1$ pandemic in a subtropical city in China, 2010-2015. Int J Infect Dis 57: 54-60.

14. Álvarez Lerma F, Marín Corral J, Vilà C, Masclans JR, Loeches IM, et al. (2017) Characteristics of patients with hospital-acquired influenza A (H1N1)pdm09 virus admitted to the intensive care unit. J Hosp Infect 95(2): 200-206

15. Mata Marín LA, Mata Marín JA, Vásquez Mota VC, Arroyo Anduiza CI, Gaytán Martínez JE, et al. (2015) Risk factors associated with mortality in patients infected with influenza A/H1N1 in Mexico. BMC Res Notes 8: 432 .

16. Mertz D, Kim TH, Johnstone J, Lam PP, Science M, et al. (2013) Populations at risk for severe or complicated in fluenza illness: systematic review and meta-analysis. BMJ 347: f5061.

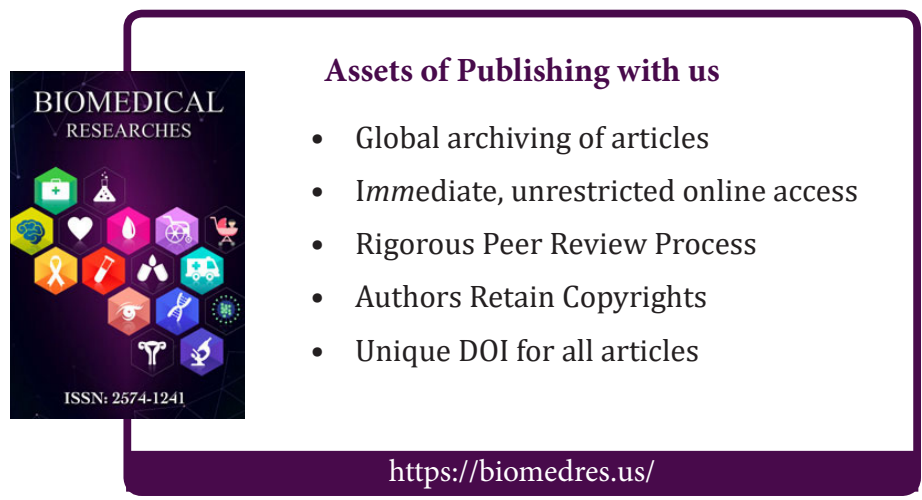

